\title{
INTERSTITIAL PNEUMONITIS SECONDARY TO INTRAVESICAL BACILLUS CALMETTE-GUERIN FOR CARCINOMA IN-SITU OF THE BLADDER
}

\author{
ERIC K. DINER, MOHAN VERGHESE \\ Department of Urology, Washington Hospital Center, Washington, DC, USA
}

\begin{abstract}
We report an 81-year-old male who developed severe interstitial pneumonitis on maintenance intravesical Bacillus Calmette-Guerin (BCG) for in-situ carcinoma of the bladder.

The patient was treated with steroids and anti-tuberculin therapy with complete response. While there is no established standard of care for the treatment of interstitial pneumonitis, recent reports describe success with combination of corticosteroids and anti-tuberculin medications.

We elected to follow this precedent and treated our patient with corticosteroids and antituberculin therapy with good outcome.
\end{abstract}

Key words: bladder neoplasms; BCG; intravesical injection; pneumonitis Int Braz J Urol. 2004; 30: 400-2

\section{INTRODUCTION}

Intravesical Bacillus Calmette-Guerin (BCG), an attenuated strain of Mycobacterium bovis, has been used in the treatment of in-situ carcinoma (CIS) and superficial carcinoma of the bladder since 1976. Its efficacy has been proven in many human trials. There has been a variety of treatment schedules and doses described with an assortment of associated side effects. (1)

We report an 81-year-old male who developed severe interstitial pneumonitis on maintenance BCG for in-situ carcinoma of the bladder. The patient was treated with steroids and anti-tuberculin therapy with complete response.

\section{CASE REPORT}

An 81-year-old man with a history of asthma and coronary artery disease was diagnosed with insitu transitional cell carcinoma of the bladder in Janu- ary 2000. He was treated with a 6-week course of BCG (Theracys) intravesically that was tolerated well. Follow up biopsies of the bladder showed no residual CIS and he was placed on maintenance BCG according to the Southwest Oncology Group protocol (2). $\mathrm{He}$ received his first three-week instillations with minimal side effects. Twenty-four hours after the first weekly dose of his second maintenance schedule, he presented to the emergency room with generalized weakness, dysuria, fever, and chills. He denied hematuria, dyspnea, chest pain, or other complaints. Upon admission, he was afebrile, with a white blood cell count (WBC) of 14.8K/UL (N 4.8-10.8 K/UL). Urine and sputum cultures showed no growth and a chest X-ray was normal. He was admitted, treated with IV antibiotics, and discharged once his WBC normalized.

Over the next 2 weeks, he developed a productive worsening cough with pleuritic chest pain, night sweats, and dyspnea at rest. He denied hemoptysis, fever, or chills. He returned to the emergency 
room where he appeared acutely ill with a temperature of 101.6 F. He was severely cyanotic, had an oxygen saturation of $86 \%$ with bilateral inspiratory rales in both lung fields. At this time, his WBC was $6.8 \mathrm{~K} / \mathrm{L}$, and urinalysis and live function tests were normal. He was readmitted to the hospital and placed in respiratory isolation due to suspected tuberculin pneumonitis.

\section{MANAGEMENT}

Initial chest $\mathrm{x}$-ray revealed granulomatous nodules in the right lower lobe (Figure-1) and a CT scan of the chest revealed diffuse interstitial fibrosis (Figure-2).

Bronchoscopy and biopsy of the lung revealed a noncaseating epithelioid granuloma while bronchial lavage showed normal alveolar and bronchial cells without acid-fast bacilli. All blood and sputum cultures revealed normal flora. He was started on triple anti-tuberculin therapy (ethambutol $1200 \mathrm{mg} \mathrm{qd}$, isonazid $300 \mathrm{mg}$ qd, rifampin $600 \mathrm{mg}$ qd) in combination with steroid therapy (prednisone $30 \mathrm{mg} \mathrm{qd}$ ).

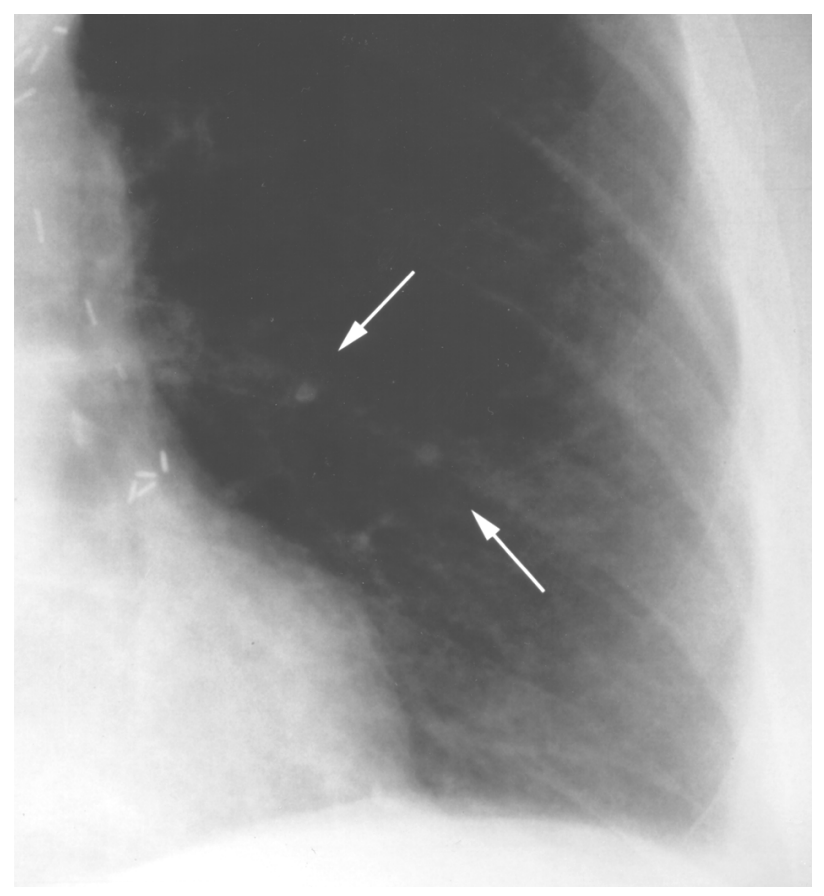

Figure 1 - Chest $x$-ray revealing granulomatous nodules in the right lower lobe (arrows).

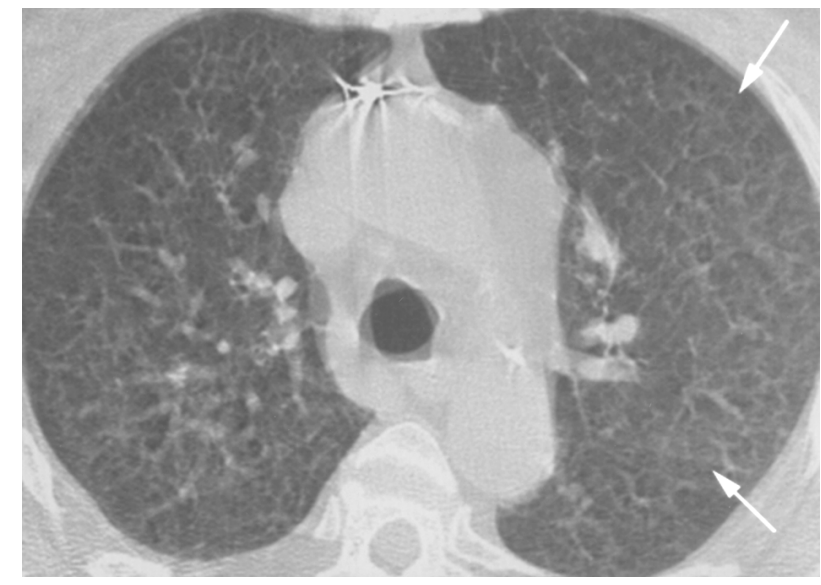

Figure 2-CT scan of the chest revealing diffuse interstitial fibrosis (arrows).

Over the next week, his pulmonary symptoms improved and he was discharged home on the ninth day. The steroids were tapered off and discontinued at 4 weeks and anti-tuberculin therapy was continued for 6 months. His follow up chest X-rays were all normal and he had no further pulmonary sequela. No further instillations of BCG were given.

\section{COMMENTS}

Intravesical BCG therapy is a relatively safe and effective therapy for superficial bladder carcinoma although up to $50 \%$ of patients can developed certain local side effects, such as fever and symptoms from cystitis. Most are self-limited and require no therapy. Other less common local genitourinary effects include granulomatous prostatitis and epididymo-orchitis, which may necessitate discontinuation of BCG therapy.

Hypersensitive systemic reactions are much less common but require immediate treatment. Fever, rash, arthritis, allergic reactions and hepatitis are all signs of hypersensity systemic reaction and possible disseminated tuberculin infection (2).

Interstitial pneumonitis is a rare complication of intravesical BCG therapy for in-situ and superficial bladder cancer. It has been described in the literature and according to one recent study, is seen in $0.7 \%$ patients following BCG therapy (2). Signs and 
symptoms can include fever, chills, dyspnea, productive cough, and hemoptosis. Diagnosis is usually made by the presence of diffuse infiltrative pattern on chest X-ray or CT scan and a positive biopsy for noncaseating epithelioid granuloma. Sputum and blood cultures are usually non-diagnostic (1).

There have been a number of case reports describing anti-tuberculin treatment for interstitial pneumonitis secondary to BCG, all based on the theory that BCG mycobacteremia and subsequent infection is the cause. A 1992 report suggests that the adverse reaction BCG may be associated with a hypersensitivity reaction and that corticosteroid use should be initiated early with anti-tuberculin therapy (3). Others have advocated the use of steroids only to treat the hypersensitivity reaction since no bacilli were cultured. While there is no established standard of care for the treatment of interstitial pneumonitis, recent reports describe success with combination of corticosteroids and anti-tuberculin medications. We elected to follow this precedent and treated our patient with corticosteroids and anti-tuberculin therapy with good outcome.

\section{REFERENCES}

1. Lamm DL, van der Meijden PM, Morales A, Brosman SA, Catalona WJ, Herr HW, et al.: Incidence and treatment of complications of bacillus Calmette-Guerin intravesical therapy in superficial bladder cancer. J. Urol. 1992; 147: 596-600.

2. Lamm DL, Blumenstein BA, Crissman JD, Montie JE, Gottesman JE, Lowe BA, et al.: Maintenance bacillus Calmette-Guerin immunotherapy for recurrent Ta, T1, and carcinoma in-situ transitional cell carcinoma of the bladder: A randomized Southwest Oncology Group study. J. Urol. 2000; 163: 1124-9.

3. Molina J, Rabian C, D'Agay M, Modai J: Hypersensitivity systemic reaction following i intravesical bacillus Calmette-Guerin: successful treatment with steroids. J. Urol. 1992; 147: 695-7.
Received: April 7, 2004

Accepted: June 20, 2004

\footnotetext{
Correspondence address:

Dr. Eric K. Diner

Washington Hospital Center

110 Irving St NW, Room 3B-19

Washington DC, 20016, USA

Fax: + 1202 877-7012

E-mail ericdiner@hotmail.com
} 